\title{
The impact of isolation on the transmission of COVID-19 and estimation of potential second epidemic in China
}

\author{
Haitao Song ${ }^{1,2}$, Fang $\mathrm{Liu}^{3,4}$, Feng $\mathrm{Li}^{3,5}$, Xiaochun $\mathrm{Cao}^{1,2}$, \\ Hao Wang ${ }^{6}$, Zhongwei Jia ${ }^{7}$, Huaiping Zhu ${ }^{8}$, Michael Y. $\mathrm{Li}^{6}$, \\ Wei $\operatorname{Lin}^{9,10}$, Hong Yang ${ }^{1,2}$, Jianghong $\mathrm{Hu}^{1,2}$, Zhen $\operatorname{Jin}^{1,2} *$ \\ ${ }^{1}$ Complex Systems Research Center, Shanxi University, Taiyuan 030006, China \\ ${ }^{2}$ Shanxi Key Laboratory of Mathematical Techniques and Big Data Analysis \\ on Disease Control and Prevention, Shanxi University, Taiyuan 030006, China \\ ${ }^{3}$ Data Science and Technology, North University of China, Taiyuan 030051, China \\ ${ }^{4}$ Department of Mathematics, Xinzhou Teachers University, Xinzhou 034000, China \\ ${ }^{5}$ College of arts and sciences, Shanxi Agricultural University, Taigu 030801, China \\ ${ }^{6}$ Department of Mathematical and Statistical Sciences, University of Alberta, Edmonton T6G 2G1, Canada \\ ${ }^{7}$ National Institute of Drug Dependence, Peking University, Beijing 100191, China \\ ${ }^{8}$ Laboratory Mathematical Parallel Systems (Lamps), Department of Mathematics and Statistics, \\ York University, Toronto M3J 1P3, Canada \\ ${ }^{9}$ Center for Computational Systems Biology, Fudan University, Shanghai 200433, China \\ ${ }^{10}$ School of Mathematical Sciences, Fudan University, Shanghai 200433, China
}

\begin{abstract}
The first case of Corona Virus Disease 2019 (COVID-19) was reported in Wuhan, China in December 2019. Since then, COVID-19 has quickly spread out to all provinces in China and over 150 countries or territories in the world. With the first level response to public health emergencies (FLRPHE) launched over the country, the outbreak of COVID-19 in China is achieving under control in China. We develop a mathematical model based on epidemiology of COVID-19, incorporating the isolation of healthy people, confirmed cases and close contacts. We calculate the basic reproduction numbers 2.5 in China (excluding Hubei province) and 2.9 in Hubei province with the initial time on January 30 which show the severe infectivity of COVID-19, and verify that the current isolation method effectively contains the transmission of COVID-19. Under the isolation of healthy people, confirmed cases and close contacts, we find a noteworthy phenomenon that is the potential second epidemic of COVID-19, and estimate the peak time and value and the cumulative number of cases. Simulations show that the isolation

*Corresponding author: Zhen Jin (jinzhn@263.net)
\end{abstract}


of close contacts tracked measure can efficiently contain the transmission of the potential second epidemic of COVID-19. With isolation of all susceptible people or all infected people or both, there is no potential second epidemic of COVID-19. Furthermore, resumption of work and study can increase the transmission risk of the potential second epidemic of COVID-19.

Keywords: COVID-19, mathematical model, basic reproduction number, potential second epidemic, isolation, close contacts

\section{Introduction}

In December 2019, the novel coronavirus pneumonia case was reported in Wuhan, China. On January 7, 2020, the novel coronavirus was first isolated by Chinese authorities [1,2]. Subsequently, the disease was named officially as Corona Virus Disease 2019 (COVID-19) by World Health Organization (WHO) [2,3]. The most important routes of transmission of COVID-19 are respiratory droplets and contact transmission, and the incubation is 2-14 days [4-6]. From the confirmed cases of COVID-19, the symptoms range from fever, cough and shortness of breath to severely ill and dying [6]. By March 18, 2020, 80,928 confirmed cases and 3245 deaths have been reported in China, where 67,800 confirmed cases and 3130 deaths are in Hubei province [7]. The COVID-19 is now threatening the public health all over the world.

China has made extraordinary efforts to contain the transmission of COVID-19 [8]. On January 20, COVID-19 is subordinated to a Class B notifiable disease by China's National Infectious Diseases Law and Frontier Health and Quarantine Law. On January 23, Chinese government started to limit movement of people in and out of Wuhan. Since January 23, the first level response to public health emergencies (FLRPHE) was gradually launched in all provinces over the country. During the FLRPHE, the government participated to deal with the public health emergency and implemented the control strategies such as limiting or stopping crowds, stopping work, class suspension, no business, management of the floating population and quarantine of transportation, adopting the emergency control methods by health administrative departments, releasing information and notification and disseminating hygienic knowledge, medical institution's quarantine or treatment on the confirmed and suspected cases, and isolation of close contacts tracked people at home. Enormous efforts have been implemented by Chinese government to prevent the spread of COVID-19. Four categories of population including confirmed cases, suspected cases, patients with fever and close contacts with confirmed case are quarantined. All confirmed cases were treated and all close contacts were tracked and quarantined [9]. Besides, China has great strengths such as community mobilization. Building and community isolation were implemented and home isolation was encouraged to prevent the transmission of COVID-19. The intervention methods implemented by China have provided 1-2 month time window for other countries to prevent their possible outbreaks.

Mathematical modelling could help us understand and control the transmission of infectious diseases [10]. Chen et al. [11] established a novel coronavirus transmission model and obtained the formula of basic reproduction number. Imai et al. [12] estimated the potential total number of COVID-19 in Wuhan. Shen et al. [13] built an ordinary differential equations (ODE) model and explored the transmission ability of diseases. Read et al. [14] used a SEIR model to assess the effect of early control methods and predict the disease development. Tang et al. [15] established an ODE model with quarantine and obtained the basic reproduction number 6.47. Zhao 
et al. [16] estimated the basic reproduction number of novel coronavirus in China using a datadriven analysis in the early phase of the outbreak. Gilbert et al. [17] evaluated the preparedness and vulnerability of African countries against their risk of importation of COVID-19 by a modelling study. However, no study has focused on the potential second epidemic of COVID-19 in China.

To assess the effect of isolation of healthy people, confirmed cases and close contacts measure on the transmission of COVID-19 in China, evaluate the peak time and value, duration and cumulative number of COVID-19 cases and estimate the potential second epidemic of COVID19, we propose a SIHR (Susceptible-Infected-Hospitalized-Removed) model incorporating the isolation of healthy people, confirmed cases and close contacts. We compute the basic reproduction number, assess effectiveness of the isolation strategy, investigate whether there is a potential second epidemic or multiple epidemics of COVID-19, estimate the peak time and value, duration and cumulative number of cases in the potential second epidemic of COVID-19 and evaluate the effect of resumption of work and study on the potential second epidemic of COVID-19.

\section{Results}

Based on the SIHR model and cumulative confirmed cases, using the least square method we estimate the parameter values $\beta, b, \gamma, \mu$ and $\alpha_{2}$ and initial values $S_{1}(0), S_{2}(0), S_{3}(0), I_{1}(0)$ and $I_{2}(0)$ which are shown in Table 1 . Here the mean absolute percentage errors (MAPE) are 0.0126 and 0.02 when the cumulative confirmed cases in China (excluding Hubei province) and in Hubei province are fitted, respectively. With the initial time on January 30, the basic reproduction numbers are 2.5 in China (excluding Hubei province) and 2.9 in Hubei province.

Under the current isolation of healthy people, confirmed cases and close contacts measure, the peak time of confirmed cases occurs around February 6 with the peak value of 5814 and disease dies out around April 5 with the cumulative number of confirmed cases of 13,340 in China (excluding Hubei province), and the peak time of confirmed cases occurs around February 15 with the peak value of 34,688 and disease dies out around May 23 with the cumulative number of confirmed cases of 69,196 in Hubei province (Fig. 2). Hence, our simulations are consistent with the real data of cumulative numbers of confirmed cases.

When isolation of healthy people, confirmed cases and close contacts measure is not implemented and parameter values and initial values are defined in Table 1, the peak time of confirmed cases is expected to appear around April 7 with the peak value of $4.099 \times 10^{8}$, and the cumulative number of confirmed cases reaches $1.271 \times 10^{9}$ in China (excluding Hubei province) (Fig. 3). This illustrates that the current isolation strategy makes the peak in advance by 60 days, and the peak value and cumulative confirmed cases are reduced by over $99 \%$. Similar results are obtained for Hubei province.

Under the current isolation of healthy people, confirmed cases and close contacts measure and parameter values and initial values are defined in Table 1, we perform simulations and find a noteworthy phenomenon which is the potential second epidemic of COVID-19 (Fig. 4). In China (excluding Hubei province), the potential second epidemic of COVID-19 will start around July 27, 2020 and persist for several years with cumulative confirmed cases of $1.58 \times 10^{8}$, and the peak time of infected population will occur around October 25, 2020 with 
the peak value of $2.29 \times 10^{7}$. In Hubei province, the second outbreak will start around July 29, 2020 and persist for several years with cumulative confirmed cases of $4.5785 \times 10^{7}$, and the peak time of infected population will occur around October 12, 2020 with the peak value of $1.1845 \times 10^{7}$. When all susceptible people or all infected people or both groups are isolated (Fig. 5), there is no potential second epidemic of COVID-19 in China (excluding Hubei province). To prevent and control the potential second epidemic of COVID-19, we investigate the effect of the isolation strategy including isolated coefficient and the time of isolation on the potential second epidemic of COVID-19 (Fig. 6). When the time of isolation for 14 days and the isolation coefficient $b=45$ of isolating close contacts tracked measure, the disease arrives to the peak of $1.428 \times 10^{7}$ around November 4 . The isolation coefficient $b=45$ delays the peak by 10 days, and the peak value is reduced by $37 \%$ in contrast with the current isolation coefficient $b=12$. When isolation coefficient $b=12$ of isolating close contacts tracked measure, the disease arrives to the peak of $1.876 \times 10^{7}$ around October 29 for the time of isolation of 33 days, and the disease arrives to the peak of $1.738 \times 10^{7}$ around October 5 for the time of isolation of 61 days which makes the peak in advance for 24 days and the peak value is reduced by $7 \%$. Similar results are obtained for Hubei province. Therefore, the isolation strategy can effectively contain the transmission of the potential second epidemic of COVID-19 by making the peak in advance and reducing the peak value.

The effect of resumption of work and study on the potential second epidemic of COVID-19 in China (excluding Hubei province) is also investigated (Figs. 7 and 8). When there is no resumption of work, the disease of potential second epidemic of COVID-19 arrives to the peak of $2.461 \times 10^{7}$ around October 18 , and the cumulative number of confirmed cases is $1.579 \times 10^{8}$. When all workers return to work and no resumption of study, the disease of potential second epidemic of COVID-19 arrives to the peak of $7.067 \times 10^{7}$ around October 31 , and the cumulative number of confirmed cases is $4.84 \times 10^{8}$. Resumption of work delays the peak by 13 days, and the peak value and cumulative confirmed cases are increased by more than twice. When there is no school, the disease of potential second epidemic of COVID-19 arrives to the peak of $7.037 \times 10^{7}$ around November 1 . When all students return to school on March 30 , the disease of potential second epidemic of COVID-19 arrives to the peak of $1.192 \times 10^{8}$ around November 24. Resumption of study delays the peak by 23 days, the peak value is increased by nearly quadruple. Similar results are obtained for Hubei province. Therefore, Resumption of work and study increases the transmission risk of the potential second epidemic of COVID-19 by delaying the peak and dramatically increasing the peak value.

\section{Discussion}

Since the first case of COVID-19 was reported in Wuhan in December, 2019, COVID-19 has spread rapidly over all provinces in China and over 150 countries, and caused 80,928 confirmed cases and 3245 deaths in China by March 18, where 67,800 reported cases and 3130 deaths are in Hubei province. According to the cumulative number of confirmed cases and the SIHR model, we estimated the related parameters value and initial values using the least square data fitting, and then we obtained the basic reproduction numbers 2.5 in China (excluding Hubei province) and 2.9 in Hubei province with the initial time January 30 in contrast with the basic reproduction numbers of SARS in Beijing $\left(R_{0}=4.91\right)$ and Hongkong $\left(R_{0}=3.6\right)$ in 2003 [18], 
and MERS in Jeddah $\left(R_{0}=3.5-6.7\right)$ in 2014 [19]. This direct comparison implies that COVID-19 is highly contagious and causes a serious threat to public health around the world.

A main contribution of our study is that isolation of healthy people, confirmed cases and close contacts measure implemented by China is highly effective to prevent and control the transmission of COVID-19. This method used in China (excluding Hubei province) makes the peak in advance by 60 days, and diminishes the peak value and cumulative confirmed cases by over $99 \%$ in contrast with the situation of no isolation. Similar results are obtained for Hubei province. The current isolation of healthy people, confirmed cases and close contacts measure effectively contains the transmission of COVID-19 in China and protects public health all over the world.

One major finding of our quantitative study is that there is a potential second epidemic of COVID-19 in China under the isolation strategy because when very few confirmed cases are diagnosed, the ignorance of isolating close contacts tracked measure makes people relax their vigilance, and infected people with mild symptoms get back to their routine work and close contacts are not quarantined, which can cause a potential second epidemic of COVID-19 [20, 21]. When all susceptible people or all infected people or both groups are isolated, there is no potential second epidemic of COVID-19. Simulations show that strengthening isolation of close contacts tracked by increasing the isolation coefficient and the time of isolation makes the peak in advance and reduces the peak value and the cumulative number of confirmed cases during the potential second epidemic of COVID-19. Numerical simulations reveal that resumption of work and study delays the peak and increases the peak value and the cumulative number of confirmed cases.

Based on the isolation of healthy people, confirmed cases and close contacts, we established the transmission dynamical model of COVID-19, calculated the basic reproduction numbers 2.5 in China (excluding Hubei province) and 2.9 in Hubei province with the initial time January 30 , which showed the severe infectivity of COVID-19 and showed that the current isolation strategy effectively contains the transmission of COVID-19 by making the peak in advance and reducing the peak value and the cumulative number of confirmed cases. Under the isolation of healthy people, confirmed cases and close contacts, we found that there may be a potential second epidemic of COVID-19, and the isolation of close contact tracked measure efficiently lowers down the probability of a potential second epidemic of COVID-19. When all susceptible people or all infected people or both groups are isolated, there is no potential second epidemic of COVID-19. In addition, resumption of work and study delays the peak and increases the peak value and the cumulative number of confirmed cases of COVID-19.

We only take the isolation of healthy people, confirmed cases and close contacts measure to prevent and control the potential second epidemic of COVID-19, and ignore the effect of vaccine on the potential second epidemic of COVID-19. We may explore such work in future when some specific vaccine becomes available. We must remain vigilant against the potential second epidemic of COVID-19 and the possibility that COVID-19 turns into a chronic epidemic disease. 


\section{Methods}

Data. Since the medical resources corresponding to patients are very different between China (excluding Hubei province) and Hubei province, then the transmission of COVID-19 in China (excluding Hubei province) and Hubei province are studied, respectively. Data of COVID-2019 cases were obtained in China from National Health Commission of the People's Republic of China and Coronavirus disease (COVID-2019) situation reports in WHO [3,22]. After the emergent closure of Wuhan city on January 23 and the whole Hubei province later, it is almost impossible to travel from Hubei province to other places. Then data were collected from January 30, 2019 to March 18, 2020 in China and in Hubei province. The data set includes the cumulative and new numbers of confirmed cases, suspected cases, death cases and cured cases, and the cumulative number of close contacts and hospitalization time.

Model. According to the 6th edition treatment of novel coronavirus pneumonia [7] and report of the WHO-China Joint Mission on COVID-19 [23], we knew that most people are susceptible for COVID-19 and the patients in the incubation period can infect healthy people. Susceptible people are infected by infected people and become infected. After the incubation period, the symptom onset and infected people become infectious. The patients go to hospital and are diagnosed. After the treatment, people are removed from hospital. We denote the susceptible people by $S$, the patients in the incubation period and the patients without being diagnosed by $I$, patients in hospital by $H$, removed people by $R$, respectively. Here the infectivity of the patients in the incubation period and the patients without being diagnosed are assumed to be the same.

After the outbreak of COVID-19 in China, FLRPHE was gradually launched in all provinces over the country since January 23. During FLRPHE, susceptible people were also advised to isolate themselves at home by isolating communities, and all close contact tracked were quarantined. Therefore, we divide susceptible people into susceptible people $\left(S_{1}\right)$, the quarantined susceptible people $\left(S_{2}\right)$ by close contacts tracked measure and general isolated susceptible people $\left(S_{3}\right)$ due to the isolation of their communities. Infected people is divided into general infected people $\left(I_{1}\right)$ including the patients in the incubation period and the patients without being diagnosed and quarantined infected people $\left(I_{2}\right)$ by close contacts tracked measure. Here we assume that all susceptible people of isolation at home cannot be infected and all infected people of isolation at home cannot infect healthy people. Based on the epidemiological pattern of COVID-19 and intervention methods [24,25], we establish the transmission dynamics of 
COVID-19 governed by the following equations:

$$
\left\{\begin{array}{l}
\frac{\mathrm{d} S_{1}(t)}{\mathrm{d} t}=\alpha_{1} S_{2}(t)-\frac{\beta S_{1}(t) I_{1}(t)}{N-S_{2}(t)-S_{3}(t)-I_{2}(t)-H(t)}-q_{1}(t)-\mu S_{1}(t)+\alpha_{2} S_{3}(t), \\
\frac{\mathrm{d} S_{2}(t)}{\mathrm{d} t}=q_{1}(t)-\alpha_{1} S_{2}(t), \\
\frac{\mathrm{d} S_{3}(t)}{\mathrm{d} t}=\mu S_{1}(t)-\alpha_{2} S_{3}(t), \\
\frac{\mathrm{d} I_{1}(t)}{\mathrm{d} t}=\frac{\beta S_{1}(t) I_{1}(t)}{N-S_{2}(t)-S_{3}(t)-I_{2}(t)-H(t)}-q_{2}(t)-\gamma I_{1}(t), \\
\frac{\mathrm{d} I_{2}(t)}{\mathrm{d} t}=q_{2}(t)-\gamma I_{2}(t), \\
\frac{\mathrm{d} H(t)}{\mathrm{d} t}=\gamma I_{1}(t)+\gamma I_{2}(t)-\delta H(t), \\
\frac{\mathrm{d} R(t)}{\mathrm{d} t}=\delta H(t)
\end{array}\right.
$$

where $q_{1}=\min \left\{S_{1}, \gamma b \frac{S_{2}}{S_{2}+I_{2}}\left(I_{1}+I_{2}\right)\right\}, q_{2}=\min \left\{I_{1}, \gamma b \frac{I_{2}}{S_{2}+I_{2}}\left(I_{1}+I_{2}\right)\right\}$. When patients go to hospital and are diagnosed $\left(\gamma\left(I_{1}+I_{2}\right)\right)$, by the close contacts tracked measure, susceptible people $\left(q_{1}\right)$ and infected people $\left(q_{2}\right)$ are quarantined by the proposition $b$. Here the numbers of quarantined susceptible people $\left(q_{1}\right)$ and quarantined infected people $\left(q_{2}\right)$ are less than the numbers of susceptible people $\left(S_{1}\right)$ and infected people $\left(I_{1}\right)$, respectively. Then $q_{1}=$ $\min \left\{S_{1}, \gamma b \frac{S_{2}}{S_{2}+I_{2}}\left(I_{1}+I_{2}\right)\right\}$ and $q_{2}=\min \left\{I_{1}, \gamma b \frac{I_{2}}{S_{2}+I_{2}}\left(I_{1}+I_{2}\right)\right\}$. After the time of isolation for 14 days $\left(1 / \alpha_{1}\right)$, the quarantined susceptible individuals become susceptible. When quarantined infected people have symptoms, they are hospitalized and diagnosed $\left(\gamma I_{2}\right)$. After the time of treatment $(1 / \delta)$, they are removed from the hospital. The communities are isolated and healthy people are also advised to isolate themselves at home unless they have something urgent to deal with. Then $\mu S_{1}$ and $\alpha_{2} S_{3}$ denote the weak movements of population from susceptible to isolated susceptible and from isolated susceptible to susceptible, respectively. Parameters and variables are defined in Table 1. The flow diagram is shown in Fig. 1.

The basic reproduction number is defined as the expected number of secondary cases produced by a single infection in a completely susceptible population, and varies as time goes on. Using the next generation matrix theory [26,27], the basic reproduction number is computed as

$$
R_{0}=\frac{\beta S_{1}(0)}{\gamma\left(N-S_{3}(0)\right)} \approx \frac{\beta}{\gamma}
$$

with the initial time January 30.

Parameter estimation. Based on the SIHR model and the cumulative number of confirmed cases, using the least square method [28] we estimate the parameter values $\beta, b, \gamma, \mu$ and $\alpha_{2}$ and initial values $S_{1}(0), S_{2}(0), S_{3}(0), I_{1}(0)$ and $I_{2}(0)$ which are shown in Table 1.

Simulation. The total populations of China and Hubei province are 1.3954 billion and 59.17 million, respectively. All close contacts tracked individuals are advised to isolate themselves at home for 14 days, then $\alpha_{1}=1 / 14$. According to the data statistics of treatment time in the provinces, we obtain that the time of treatment is about 10.534 days outside Hubei province and 18 days inside Hubei province, then $\delta=1 / 10.534$ on provinces outside Hubei and $\delta=1 / 18$ 
in Hubei province. We choose the cumulative number of confirmed cases from January 30 to March 18 in China (excluding Hubei province). The cumulative number of confirmed cases from January 30 to February 4 and from February 12 to March 18 are chosen in Hubei province since comprehensive troubleshooting was carried out from February 8 to February 11 in Wuhan and caused the exception data. By January 30, 2020 in China (excluding Hubei province), 3886 confirmed cases were reported leading to $H(0)=3886$, and 65 patients were removed from hospital leading to $R(0)=65$. By January 30, 2020 in Hubei province, 5806 confirmed cases were reported leading to $H(0)=5806$, and 320 patients were removed from hospital leading to $R(0)=320$. We estimate other parameters and initial values using the least square method in Table 1. All numerical simulations are carried out using Matlab programs.

\section{References}

[1] World Health Organization (WHO). Novel Coronavirus-China. https:/www.who.int/csr/don/12-january-2020-novel-coronavirus-china/en/. [2020-01-12]

[2] Zhu, N. et al. A novel coronavirus from patients with pneumonia in China, 2019. New England Journal of Medicine (2020).

[3] World Health Organization (WHO). Coronavirus disease (COVID-2019) situation reports. https://www.who.int/emergencies/diseases/novel-coronavirus-2019/situationreports/[Accessed 12 February 2020].

[4] Guan, W. et al. Clinical characteristics of 2019 novel coronavirus infection in China. MedRxiv (2020).

[5] Huang, C. et al. Clinical features of patients infected with 2019 novel coronavirus in Wuhan, China. The Lancet (2020).

[6] Centers for Disease Control and Prevention. Coronavirus Disease 2019 (COVID-19). https://www.cdc.gov/coronavirus/2019-ncov/index.html.

[7] Chinese Center for Disease Control and Prevention. http://2019ncov.chinacdc.cn/2019$\mathrm{nCoV} /$.

[8] Frieden, T. A Strong Public Health System: Essential for Health and Economic Progress. China CDC Weekly 2, 128-130 (2020).

[9] Tu, W. et al. Epidemic Update and Risk Assessment of 2019 Novel Coronavirus-China, January 28, 2020. China CDC Weekly 2, 83-86 (2020).

[10] Egger, M. et al. Developing WHO guidelines: time to formally include evidence from mathematical modelling studies. F1000Research 6 (2017).

[11] Chen, T. et al. A mathematical model for simulating the transmission of Wuhan novel Coronavirus. bioRxiv (2020). 
[12] Imai, N. et al. Estimating the potential total number of novel Coronavirus (2019-nCoV) cases in Wuhan City, China. Preprint published by the Imperial College London (2020).

[13] Shen, M. et al. Modelling the epidemic trend of the 2019 novel coronavirus outbreak in China. bioRxiv (2020).

[14] Read, J. M. et al. Novel coronavirus 2019-nCoV: early estimation of epidemiological parameters and epidemic predictions. medRxiv (2020).

[15] Tang, B. et al. Estimation of the Transmission Risk of the 2019-nCoV and Its Implication for Public Health Interventions. Journal of Clinical Medicine 9, 462 (2020).

[16] Zhao, S. et al. Preliminary estimation of the basic reproduction number of novel coronavirus (2019-nCoV) in China, from 2019 to 2020: A data-driven analysis in the early phase of the outbreak. International Journal of Infectious Diseases (2020).

[17] Gilbert, M. et al. Preparedness and vulnerability of African countries against importations of COVID-19: a modelling study. The Lancet (2020).

[18] Gumel, A. B. et al. Modelling strategies for controlling SARS outbreaks. Proceedings of the Royal Society of London. Series B: Biological Sciences 271, 2223-2232 (2004).

[19] Majumder, M. S.et al. Estimation of MERS-coronavirus reproductive number and case fatality rate for the spring 2014 Saudi Arabia outbreak: insights from publicly available data. PLoS Currents 6, (2014).

[20] Li, R. et al. Substantial undocumented infection facilitates the rapid dissemination of novel coronavirus (SARS-CoV2). Science, 2020.

[21] Elisabeth, M. Covid-19: UK starts social distancing after new model points to 260000 potential deaths BMJ 368, m1089 (2020).

[22] National Health Commission of the People's Republic of China. http://www.nhc.gov.cn/xcs/xxgzbd/gzbd index.shtml [2020-02-12].

[23] World Health Organization (WHO). Coronavirus disease (COVID-2019) outbreak, report of the WHO-China Joint Mission on COVID-19. https://www.who.int/docs/defaultsource/coronaviruse/who-china-joint-mission-on-covid-19-final-report.pdf.

[24] Lu, R. et al. Genomic characterisation and epidemiology of 2019 novel coronavirus: implications for virus origins and receptor binding. The Lancet (2020).

[25] Yang, Y. et al. Epidemiological and clinical features of the 2019 novel coronavirus outbreak in China. medRxiv (2020).

[26] Diekmann, O., Heesterbeek, J. A. P. \& Metz, J. A. J. On the definition and the computation of the basic reproduction ratio $R_{0}$ in models for infectious diseases in heterogeneous populations, J. Math. Biol. 28, 365382 (1990). 
[27] Van den Driessche, P. \& Watmough, J. Reproduction numbers and sub-threshold endemic equilibria for compartmental models of disease transmission. Mathematical biosciences 180, 29-48 (2002).

[28] Least Squares Method. https://www.investopedia.com/terms/1/least-squares-method.asp.

\section{Acknowledgments}

The research is supported by the National Natural Science Foundation of China (No. 11601291, 61873154, U1611264).

\section{Author contributions}

HS, ZJ and FL derived the SIHR model and designed the study. HS, HY, FL and JH carried out the data analysis, some numerical simulations and the parameter estimations. HS, ZJ and HW provided the literature review and writing. All authors contributed to the reviewing of the manuscript.

\section{Competing interests}

All authors declare that they have no competing interests.

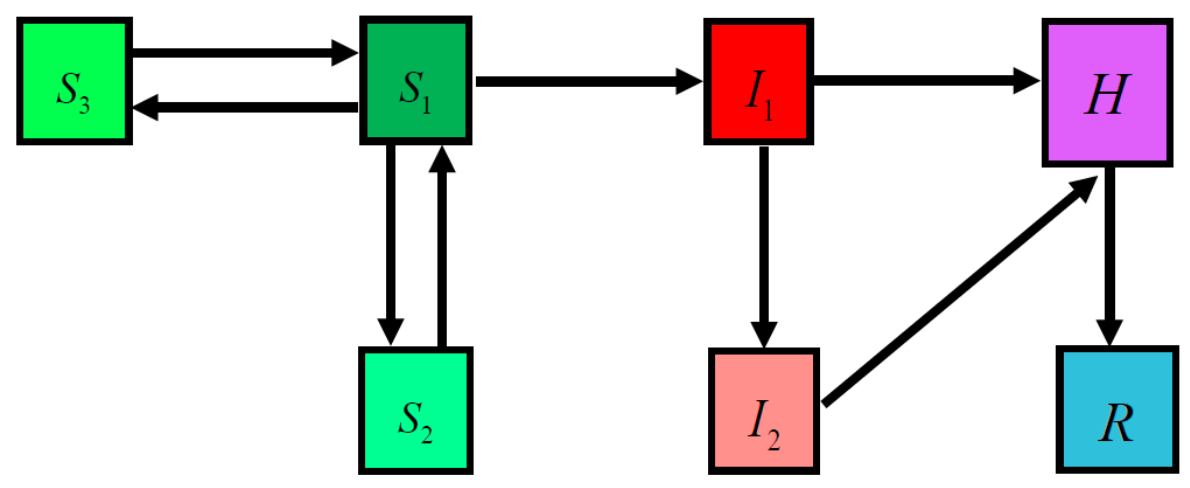

Figure 1: The flow diagram of COVID-19 in SIHR model. 
Table 1: Related parameters and initial values in China (excluding Hubei province) and Hubei province.

\begin{tabular}{|c|c|c|c|}
\hline & $\begin{array}{l}\text { Related parameters and initial values in China } \\
\text { (excluding Hubei province) }\end{array}$ & & \\
\hline Parameter & Descriptions & Mean value & Source \\
\hline$\alpha_{1}$ & $\begin{array}{l}\text { The time of isolation at home } \\
\text { for susceptible population }\end{array}$ & $1 / 14$ & [22] \\
\hline$\beta$ & The transmission rate of COVID-19 & 0.3567 & Estimated \\
\hline$b$ & The isolation coefficient & 12 & Estimated \\
\hline$\gamma$ & The hospitalization rate of infected population & 0.1429 & Estimated \\
\hline$\delta$ & The discharged rate from hospital & 0.0949 & Data \\
\hline$\mu$ & $\begin{array}{l}\text { The transfer rate from susceptible to } \\
\text { isolated susceptible population }\end{array}$ & $1.76 \times 10^{-4}$ & Estimated \\
\hline$\alpha_{2}$ & $\begin{array}{l}\text { The transfer rate from isolated susceptible } \\
\text { to susceptible population }\end{array}$ & $5.05 \times 10^{-6}$ & Estimated \\
\hline Initial Values & Descriptions & Mean value & Source \\
\hline$N$ & $\begin{array}{l}\text { Total population of China } \\
\text { (excluding Hubei province) }\end{array}$ & $1.3362 \times 10^{9}$ & Estimated \\
\hline$S_{1}(0)$ & Initial susceptible population & $2.6723 \times 10^{8}$ & Estimated \\
\hline$S_{2}(0)$ & Initial quarantined susceptible population & 3762 & Estimated \\
\hline$S_{3}(0)$ & Initial isolated susceptible population & $1.069 \times 10^{9}$ & Estimated \\
\hline$I_{1}(0)$ & Initial infected population & 4101 & Estimated \\
\hline$I_{2}(0)$ & Initial quarantined infected population & 700 & Estimated \\
\hline$H(0)$ & Initial hospitalized population & 3886 & Data \\
\hline \multirow[t]{2}{*}{$R(0)$} & Initial removed individuals & 64 & Data \\
\hline & Related parameters and initial values in Hubei & & \\
\hline Parameter & Descriptions & Mean value & Source \\
\hline$\alpha_{1}$ & $\begin{array}{l}\text { The time of isolation at home } \\
\text { for susceptible population }\end{array}$ & $1 / 14$ & {$[22]$} \\
\hline$\beta$ & The transmission rate of COVID-19 & 0.3999 & Estimated \\
\hline$b$ & The isolated coefficient & 5 & Estimated \\
\hline$\gamma$ & The hospitalization rate of infected population & 0.1379 & Estimated \\
\hline$\delta$ & The discharged rate from hospital & $1 / 18$ & Data \\
\hline$\mu$ & $\begin{array}{l}\text { The transfer rate from susceptible to } \\
\text { isolated susceptible population }\end{array}$ & $9.983 \times 10^{-6}$ & Estimated \\
\hline$\alpha_{2}$ & $\begin{array}{l}\text { The transfer rate from isolated susceptible } \\
\text { to susceptible population }\end{array}$ & $4.825 \times 10^{-5}$ & Estimated \\
\hline Initial Values & "Descriptions & Mean value & Source \\
\hline$N$ & Total population of Hubei province & $5.917 \times 10^{7}$ & Estimated \\
\hline$S_{1}(0)$ & Initial susceptible population & $1.18 \times 10^{7}$ & Estimated \\
\hline$S_{2}(0)$ & Initial quarantined susceptible population & 5367 & Estimated \\
\hline$S_{3}(0)$ & Initial isolated susceptible population & $4.7336 \times 10^{7}$ & Estimated \\
\hline$I_{1}(0)$ & Initial infected population & 12973 & Estimated \\
\hline$I_{2}(0)$ & Initial quarantined infected population & 2023 & Estimated \\
\hline$H(0)$ & Initial hospitalized population & 5806 & Data \\
\hline$R(0)$ & Initial removed individuals & 320 & Data \\
\hline
\end{tabular}



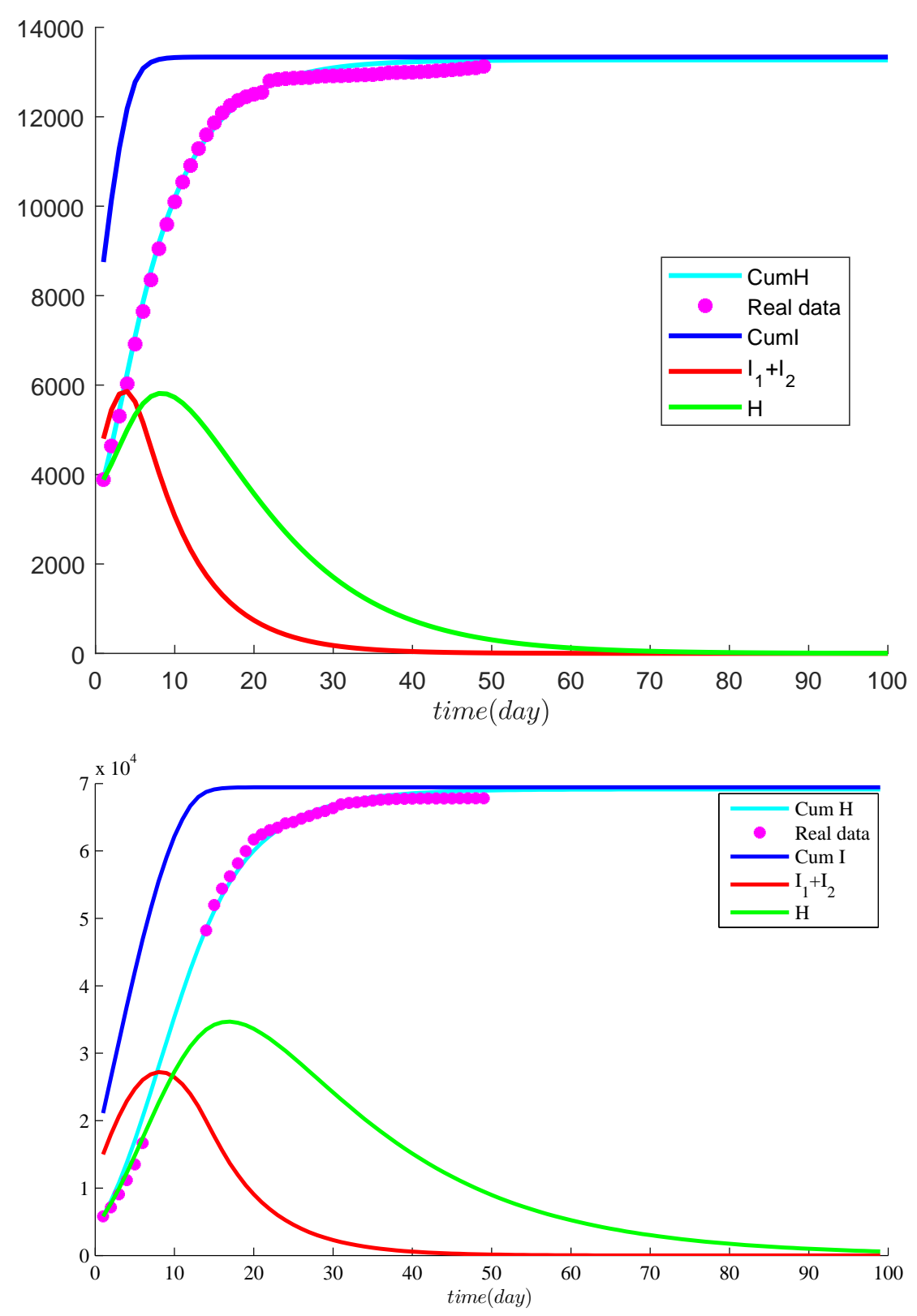

Figure 2: The cumulative number of confirmed cases and infected cases in China (excluding Hubei province) and Hubei province are shown under the current intervention methods. Where $\mathrm{CumH}$ denotes the cumulative number of confirmed cases, CumI is cumulative number of infected population, $I_{1}+I_{2}$ is the infected population and $H$ represents the confirmed cases. Other parameter values and initial values are defined in Table 1. 

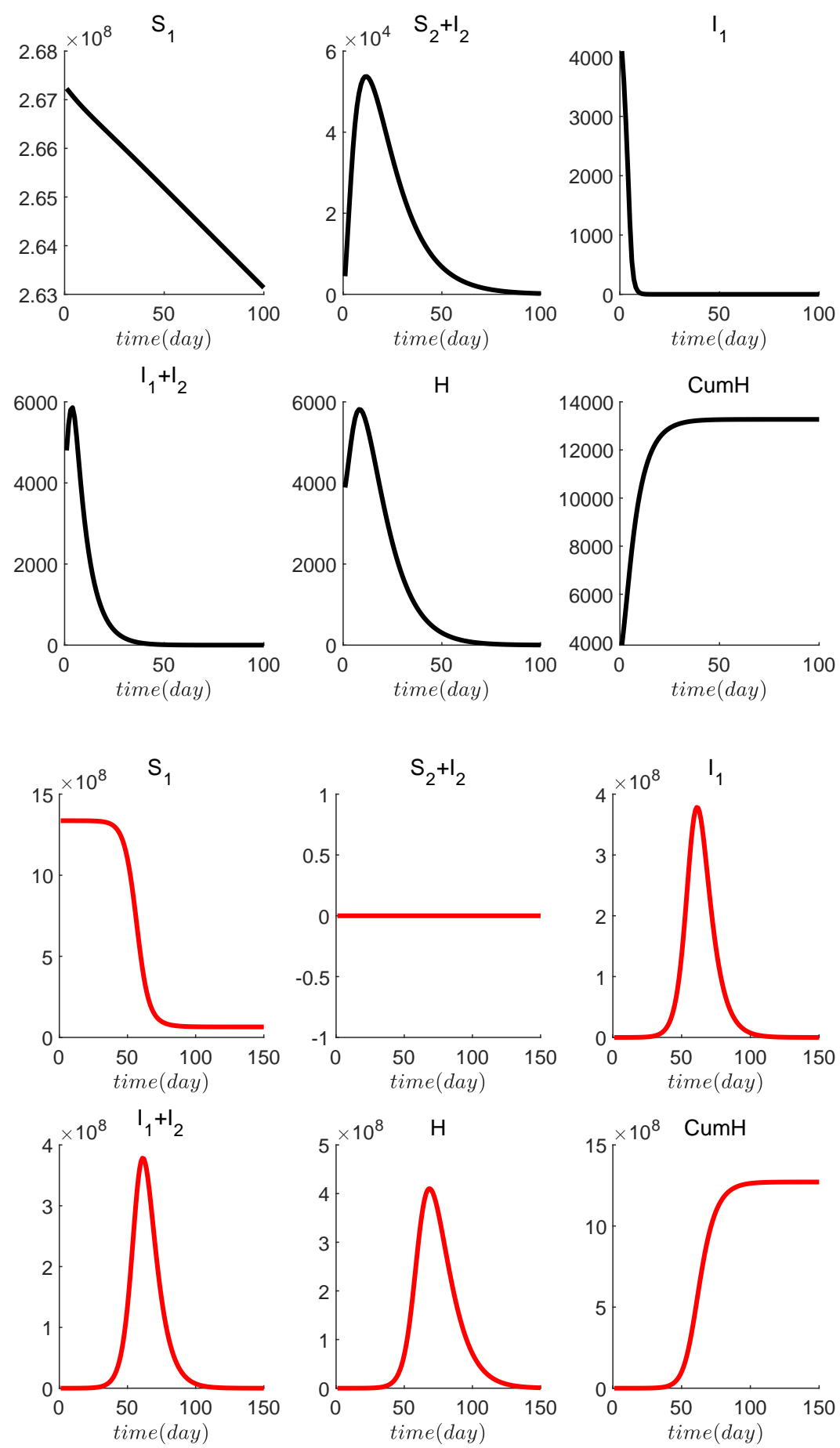

Figure 3: The effect of the isolation of healthy people, confirmed cases and close contacts measure on the transmission of COVID-19 in China (excluding Hubei province). The top figure shows the cumulative number of confirmed cases and confirmed cases under the current intervention methods. The bottom figure shows the cumulative number of confirmed cases and confirmed cases when the current isolation strategy is not carried out. Where CumH denotes the cumulative number of confirmed cases, CumI is cumulative number of infected population, $S_{2}+I_{2}$ is the quarantined susceptible and infected population, $I_{1}+I_{2}$ is the all infected population and $H$ represents the confirmed cases. Other parameter values and initial values are defined in Table 1. 

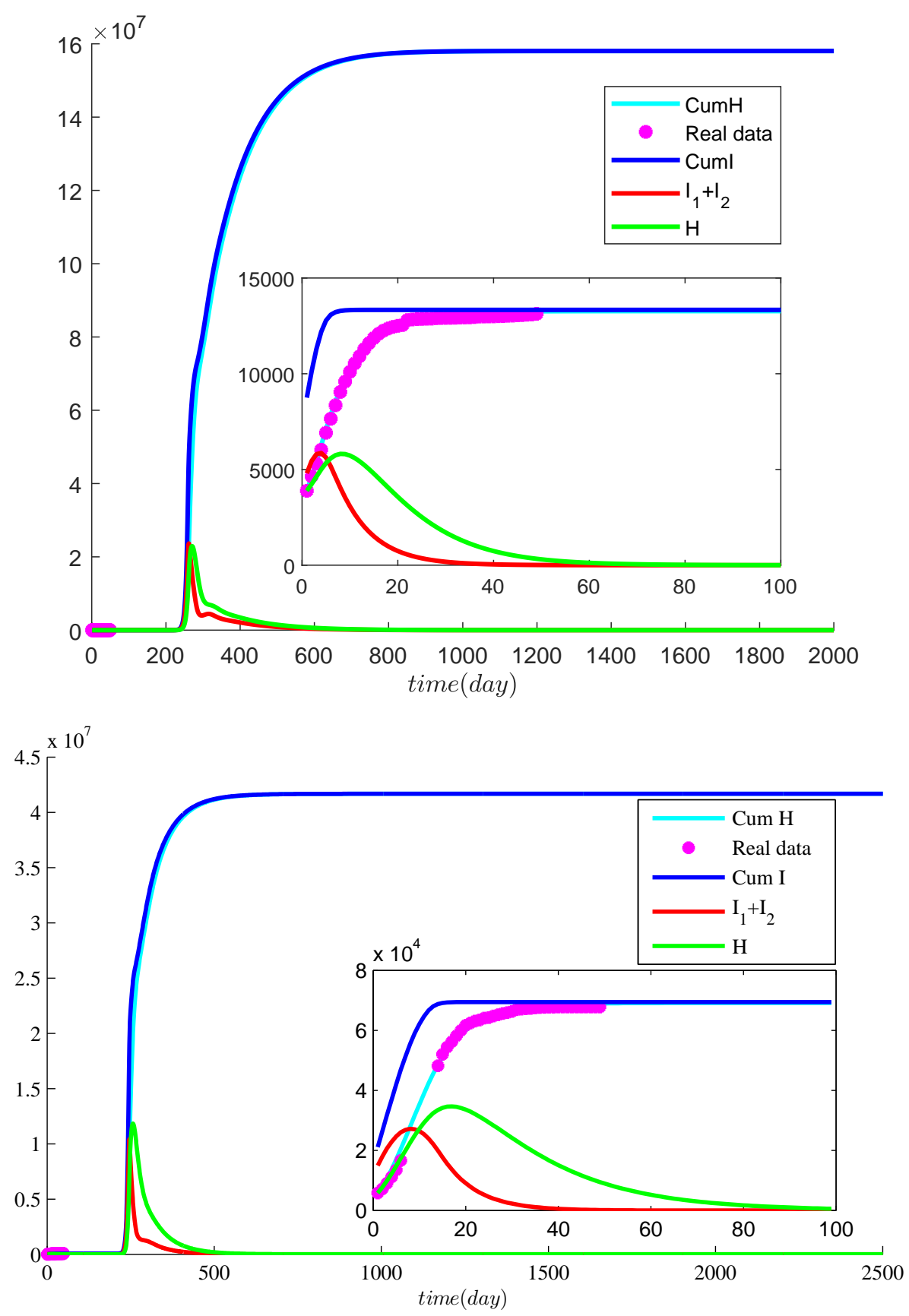

Figure 4: The predicted cumulative number of confirmed cases and infected cases of the potential second epidemic of COVID-19 in China (excluding Hubei province) and Hubei province are shown under the current intervention methods. The embedded figures are the thumbnail of Fig. 2. Where CumH denotes the cumulative number of confirmed cases, CumI is cumulative number of infected population, $I_{1}+I_{2}$ is the infected population and $H$ represents the confirmed cases. Other parameter values and initial values are defined in Table 1. 

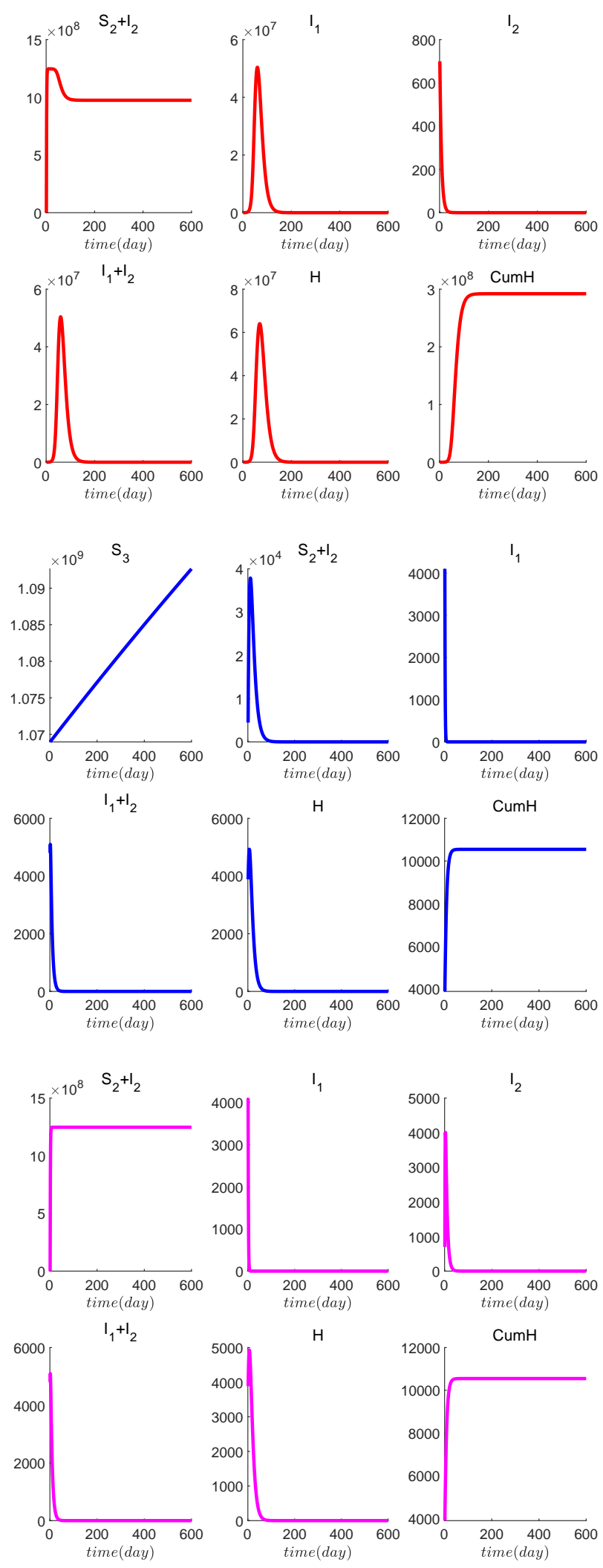

Figure 5: There is no second epidemic in China (excluding Hubei province) when all susceptible people (top) or all infected people (middle) or all susceptible people and infected people (bottom) are isolated. Where $C u m H$ denotes the cumulative number of confirmed cases, CumI is cumulative number of infected population, $1 S_{2}+I_{2}$ is the quarantined susceptible and infected population, $I_{1}+I_{2}$ is the all infected population and $H$ represents the confirmed cases. Parameter values and initial values are defined in Table 1. 

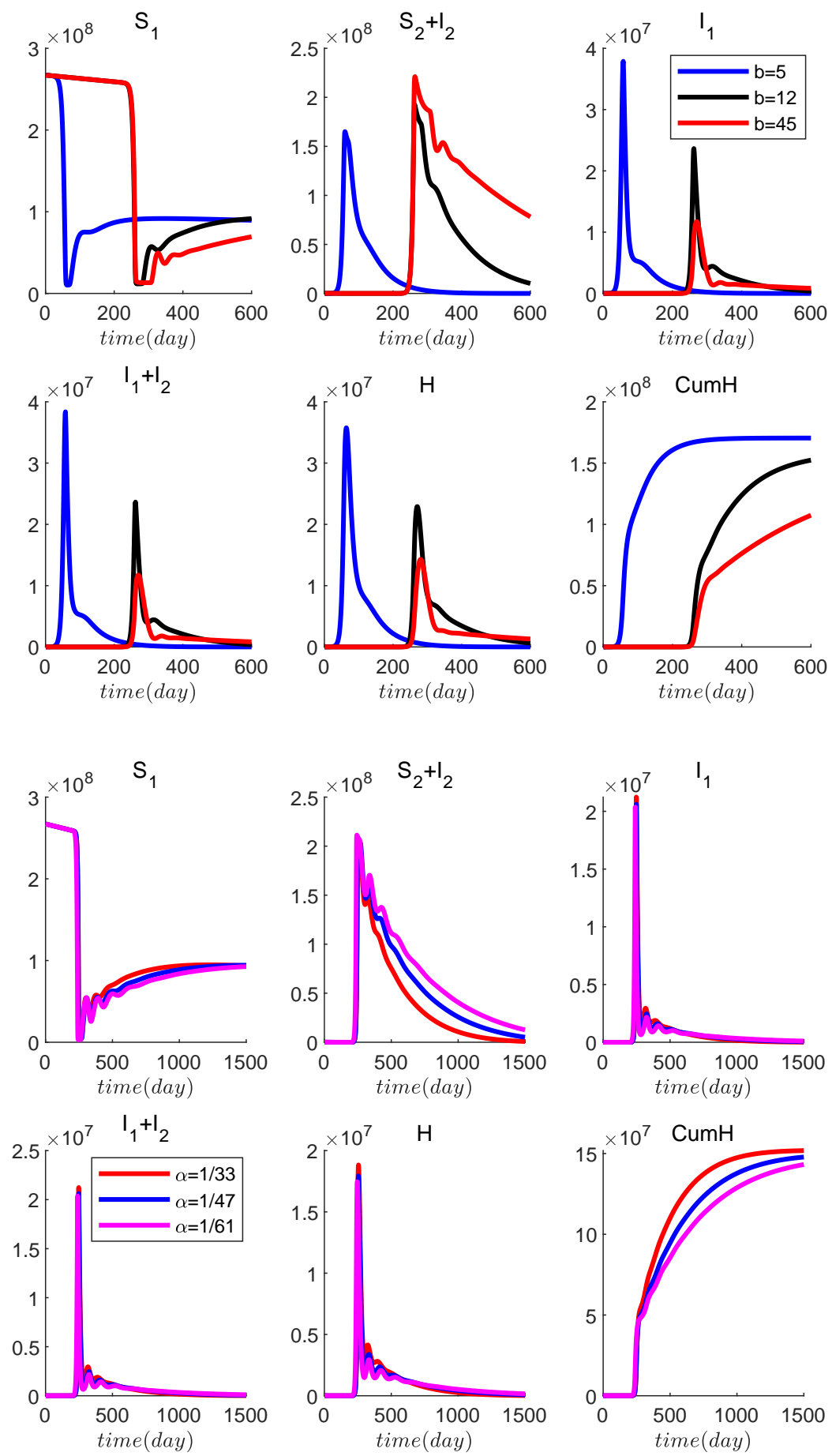

Figure 6: Under the current intervention methods, the top figure shows the effect of isolated coefficient $b$ of close contacts tracked on the potential second epidemic of COVID-19 when the time of isolation is 14 days, and the bottom figure shows the effect of the time of isolation on the potential second epidemic of COVID-19 when the isolated coefficient $b$ of close contacts tracked is 12 . Here we assumed that $80 \%$ of the susceptible population are isolated in China (excluding Hubei province) on January 30. Where $\mathrm{CumH}$ denotes the cumulative number of confirmed cases, CumI is cumulative number of infected population, $S_{2}+I_{2}$ is the quarantined susceptible and infected population, $I_{1}+I_{2}$ is the all infected population and $H$ represents the confirmed cases. Parameter values and initial valaes are defined in Table 1. 

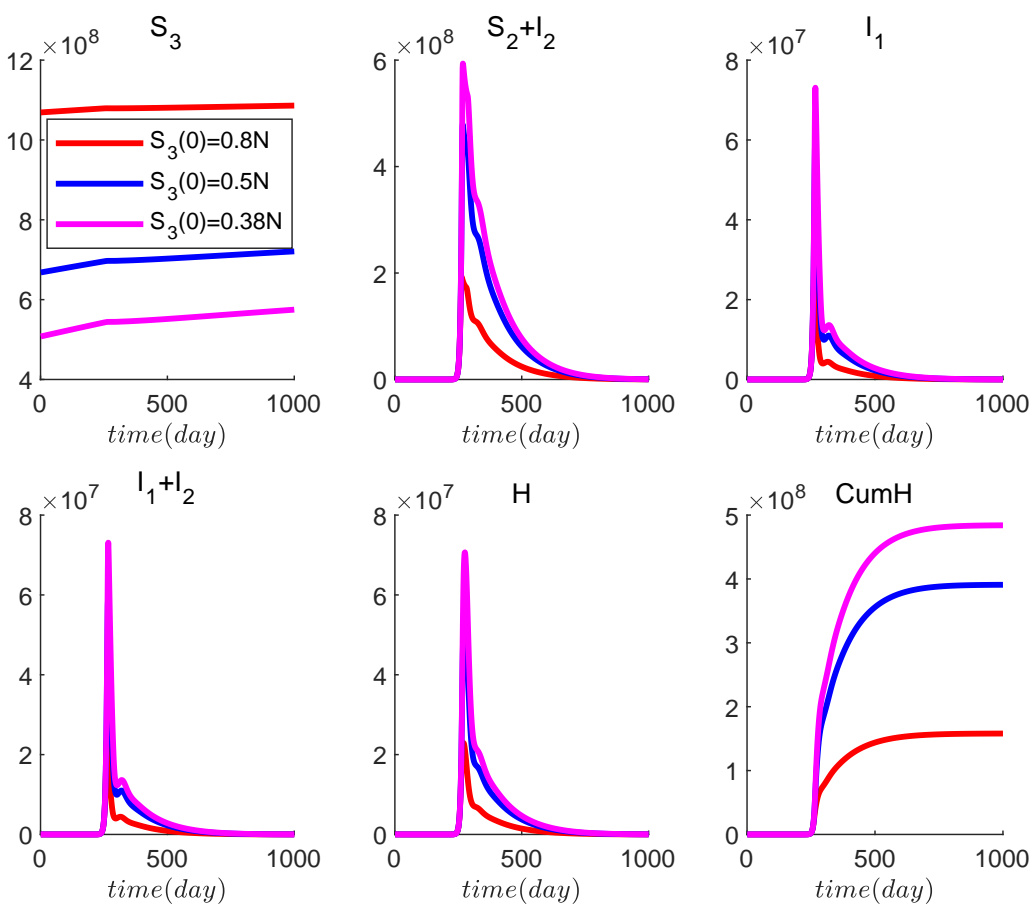

Figure 7: The effect of resumption of work on the potential second epidemic of COVID-19 in China (excluding Hubei province) is assessed under the isolation strategy. Where $0.8 \mathrm{~N}, 0.5 \mathrm{~N}$ and $0.38 \mathrm{~N}$ denote that $80 \%, 50 \%$ and $38 \%$ of the susceptible population are isolated on January 30, respectively. Where $C u m H$ denotes the cumulative number of confirmed cases, CumI is cumulative number of infected population, $S_{2}+I_{2}$ is the quarantined susceptible and infected population, $I_{1}+I_{2}$ is the all infected population and $H$ represents the confirmed cases. Other parameter values and initial values are defined in Table 1. 

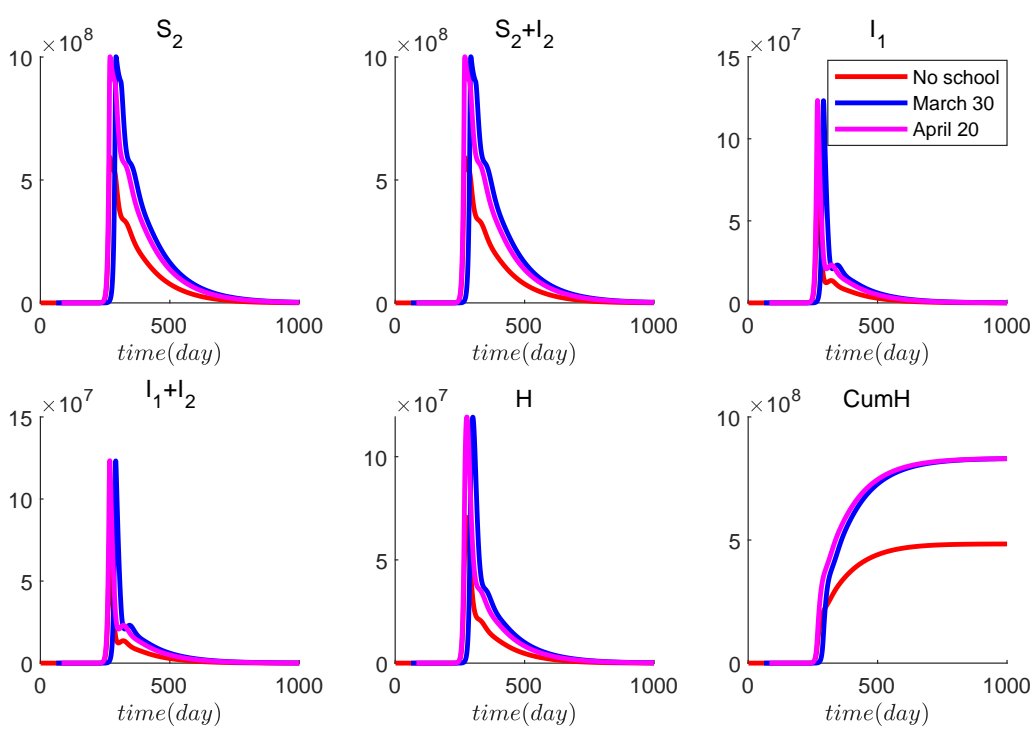

Figure 8: Under the isolation strategy, the effect of resumption of study on the potential second epidemic of COVID-19 in China (excluding Hubei province) is assessed in case of no school, resumption of study on March 30 and April 20. We assume that 38\% of the susceptible population are isolated before school. Here, $\mathrm{CumH}$ denotes the cumulative number of confirmed cases, CumI is cumulative number of infected population, $S_{2}+I_{2}$ is the quarantined susceptible and infected population, $I_{1}+I_{2}$ is the all infected population and $H$ represents the confirmed cases. Other parameter values and initial values are defined in Table 1. 